\title{
Redistribution of Energy in Electromagnetic Wave Interactions Interference of electromagnetic waves; a different approach
}

\author{
C.K.G. Piyadasa ${ }^{\dagger}$ \\ Department of Physics, University of Colombo, Colombo 00300, Sri Lanka
}

\begin{abstract}
One of the well-known effects of electromagnetic waves is interference. Redistribution of energy in interference is well documented in literature but the mechanism has not been discussed in detail. A set of new experiments has been designed and conducted to observe the actual redistribution of the energy of the electromagnetic waves while being interfered and after leaving the region of interference.
\end{abstract}

In this paper, it is shown that the redistribution of energy in interference of electromagnetic waves maintains the energy distribution prevailed at the moment they leave the disturbance (interference) area. Modified wave fronts which separated after intersection of two coherent waves in microwave frequency is demonstrated. Results are presented in pictorial and graphical models in order to understand the phenomena,. Further experiments are underway and possible mechanism will be discussed later.

\section{INTRODUCTION}

One of the unresolved problems in physics is wave-particle duality of electromagnetic (EM) waves. It is known that certain physical phenomena can be explained only using particle nature of EM waves $[1,2]$ while the other phenomena can be explained only using their wave nature [3]. For example, photoelectric effect is described by the particle nature

\footnotetext{
${ }^{\dagger}$ E-mail: gaminickg@yahoo.com
} 
of EM waves and diffraction and interference are explained by the wave nature. The wave nature was first postulated by $\mathrm{C}$. Huygens and he described that light propagates as a wavefront [4]. He further explained that at any given instant, each point on the wavefront is the origin of a secondary wave which propagates outwards as a spherical wave. The secondary waves then combine to form a new wavefront. In a monochromatic system, the time variation of optical disturbance, $A$ (magnitude of electric vector $|\mathrm{E}|$ ) can be written as

$$
A=A_{0} e^{j(\omega t+\phi)}
$$

where $A_{0}$ is the maximum amplitude, $\omega$ is the angular frequency and $\phi$ is the phase angle. This is true for all EM waves. One of the key features of EM wave nature is superposition. Superposition of two waves $A_{1}=A_{10} e^{j\left(\omega t+\phi_{1}\right)}$ and $A_{2}=A_{20} e^{j\left(\omega t+\phi_{2}\right)}$ is given by

$$
A=A_{1}+A_{2}=A_{10} e^{j\left(\omega t+\phi_{1}\right)}+A_{20} e^{j\left(\omega t+\phi_{2}\right)}
$$

where $A_{10}, A_{20}$ are maximum amplitudes and $\phi_{1}, \phi_{2}$ are phases of respective waves Intensity, $I$ of a EM wave is defined as the time average of $A$ and $I \propto\langle A\rangle^{2}$. The intensity (average power per unit area) is the measure of energy

$I=\frac{1}{2 \mu_{0} c} A_{0}^{2}$

where

$$
A_{0}^{2}=A_{10}^{2}+A_{20}^{2}+2 A_{10} A_{20} \operatorname{Cos}\left(\phi_{1}-\phi_{2}\right)
$$

where $\mu_{0}$ is the permeability of free space and $c$ is the speed of light. From eq. 3 and eq. 4 , the resultant intensity $(I)$ of two waves with intensities $I_{1}$ and $I_{2}$ is given by $I=I_{1}+I_{2}+2 \sqrt{I_{1} I_{2}} \operatorname{Cos}\left(\phi_{1}-\phi_{2}\right)$

If $I_{1}=I_{2}$ and $\phi_{1}=\phi_{2}$ (zero phase difference)

$$
I=4 I_{1}
$$


Whenever the phase difference is $180^{\circ}$ the intensity becomes zero.

$I=0$

Young's double slit experiment (1802) [3] was one of the landmark experiments to determine the wave nature of light (Fig. 1(a)). It describes the superposition of two coherent light waves; intensities of the waves with same phase (in-phase) add up as in Eq. 6 and produce bright spots or in other words cause constructive interference. Waves that are $180^{\circ}$ out of phase will create dark regions (Eq. 7) or cause destructive interference. These regions are also referred to as "maxima" (bright, maximum intensity $4 I_{1}$ ) and "minima" (dark, zero intensity) respectively. After Young's experiment, several interferometers were developed in order to demonstrate the principle. A.A. Michelson (1882)[5], Fabry and Perot (1899)[6], Twyman and Green[7] and Mach and Zehnder[8] were some of them. There are several practical ways to produce two overlapping light beams from a single source such as Fresnal's biprism [9], Fresnel's double mirror[10] and Loyd's mirror[11].

If $\lambda$ is the wavelength, $d$ is the distance between two slits and $L$ is the distance to the screen from the slits, the general condition for a next bright area, $y$ is given by following mathematical relation (see Fig. 1(a))

$y \approx \frac{m \lambda L}{d}$

where $\mathrm{m}=0,1,2 \ldots \ldots$

This is an important result applicable to all interference and diffraction effects where two primary coherent monochromatic sources are nearly parallel and predicts energy redistribution in space. Fig. 1(b) shows this energy (intensity) redistribution across an interference pattern on a screen. A single beam of light gives a uniform distribution of intensity, $I_{I}$ throughout the screen as shown in curve $I$ of Fig. 1(b). Two non-coherent beams of equal intensities $I_{l}$ would yield a uniformly illuminated screen with an intensity $2 I_{1}$. See curve $I I$ in Fig. 1(b). If the two waves are coherent, or in other words, the two beams have a phase relationship that satisfies the condition for interference in Eq. (9), they form alternative maxima and minima, hence energy redistribution. If initial amplitudes of the two coherent beams are equal, then the maxima are four times the intensity of the 
individual contribution as shown in curve III of Fig. 1(b). However, the area under interference curve $I I I$ is equal to that of the curve $I I$.

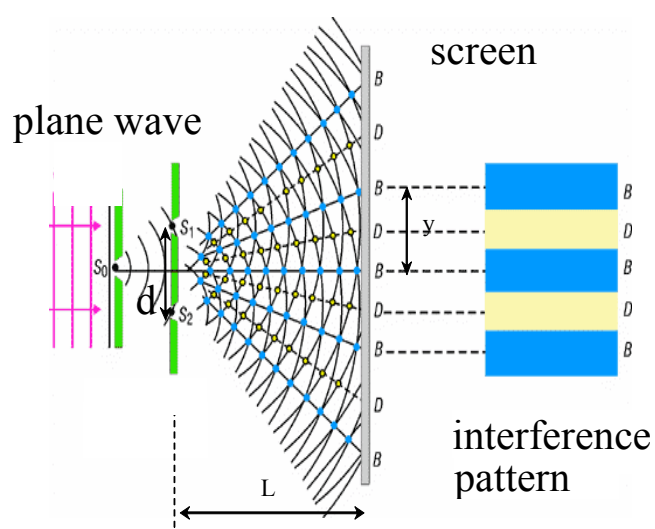

(a)

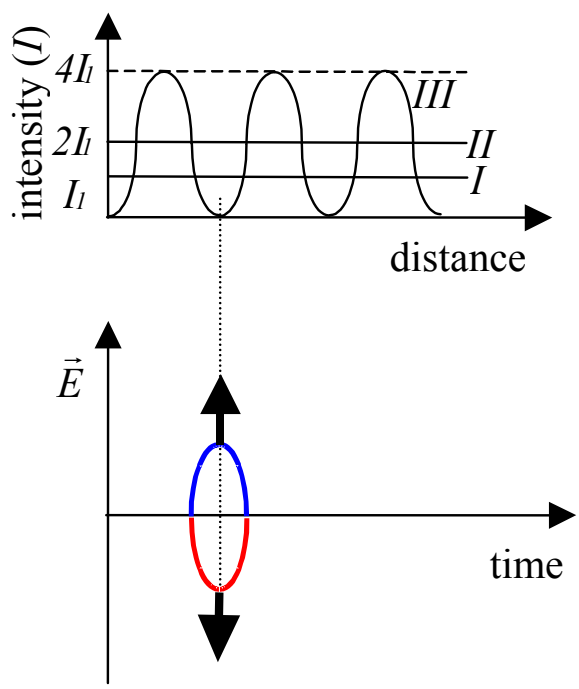

Figure 1: (a) Young's double slit experiment. $S_{0}, S_{1}$ and $S_{2}$ are fine slits and act as secondary point sources of light. Plane wave of light incident at slit $S_{0}$. " $\boldsymbol{B}$ " indicates bright areas and " $\boldsymbol{D}$ " indicates dark areas. The condition for interference pattern is $y=\frac{m \lambda L}{d}$ where $\lambda$ is the wavelength of incident wave, $L$ is the distance between plane of the slits and the screen and $d$ is the distance between two slits. (b) Intensity distributions produced by light beams: a single light beam (I), double incoherent light beams (II) and double coherent light beams (III). (c) equal and opposite $\vec{E}$ vectors of two beams produces a null at interference pattern.

This leads to a following contradiction with the existing wave theory. According to the wave theory, energy of a EM wave is associated with the electrical vector $\vec{E}$ and is proportional to $\frac{1}{2} \varepsilon_{0} E_{0}^{2}$. However if we consider zero nodes in a interference pattern, to construct destructive interference or zero intensity, electrical vectors of two waves should be equal and opposite at that particular point Fig. (1(c)). In other words that can be considered as Tug of War! If two forces are equal then there is no resultant movement in the rope.

At the same time, maxima carry an intensity which is four times the original intensity when both beams are coherent and identical in amplitudes. Although this is explained mathematically through an addition of eq. 4 and 5, it seems that the energy of minima has been shifted towards maxima. Since the two $\vec{E}$ vectors with equal amplitudes have to be present at minima to ensure zero intensities at those regions, the classical wave theory 
leads to an important question, whether the energies dissociate themselves from their $\vec{E}$ vectors at such minima.

Furthermore, if the classical theory is true, redistribution of energy occurs only in the region where two coherent EM waves cross over, and each beam should regain their initial features that were in existence prior to the interference, as soon as the two beams leave the cross over zone. This is one of the primary conclusions that can be drawn from the wave theory.

\section{EXPERIMENTAL APPROACH}

The main objective of the present investigation is to measure energy distribution of interference quantitatively. The first step of the study was to re-investigate the energy redistribution due to the interference and confirm the validity of the eq. 6 above. Interference of light can be observed easily with the naked eye in a simple experiment, and therefore a Michelson interferometer (Fig. 2(a)), one of the most common instruments in students labs together with readily available $\mathrm{He}-\mathrm{Ne}$ laser light, was used at the initial part of this investigation. A photo diode was used to scan and measure the energy distribution of the interference pattern. A high speed (FFD-040) photo diode (active area $0.9 \times 0.9 \mathrm{~mm}^{2}$ ) was used as an energy detector. The current flowing in the photo diode is linearly proportional to the intensity, and hence proportional to the incident power of the EM wave. The diode and the amplifier were mounted on a stepper motor driven platform (Aerotech, ATS100-50-20) and scanning was done using in-house developed software via the interface (Simple step, XY microxxA).

Non-localized rectilinear fringes (Fig. 2(b)) were obtained using Michelson interferometer. A He-Ne laser (632.8 $\mathrm{nm}$ Scientific Laser SLC.8mw CLR) was used as the monochromatic source of light and the interferometer was set-up as in Fig. 2(a). The photo diode linearly scans the screen with $62.16 \mu \mathrm{m}$ steps (740 steps for $46 \mathrm{~mm}$ ) and for each increment the voltage reading was recorded in a data file. Number of vertical scans was done perpendicular to the rectangular fringes. Average intensity values of these were plotted, and are shown in Fig. 2(c). Intensities of two light beams from mirrors 1 (M1) and 2 M(2) were measured separately. Mirrors were covered one at a time to obtain these measurements, and the results are shown in Fig. (2(c)), as curves $I$ (red) and $I I$ (blue). The 
curve $I I I$ (green) depicted the algebraic sum of curves $I$ and $I I$. The interference pattern (when both mirrors were unblocked) is shown in curve $I V$ (black). The measured ambient noise level was 0.005 (arbitrary units)

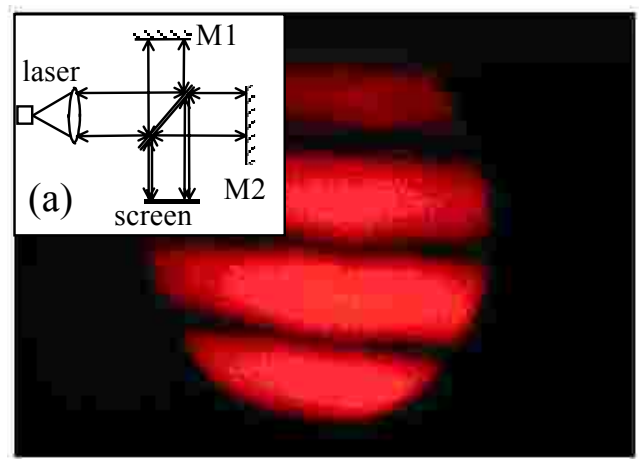

(b)

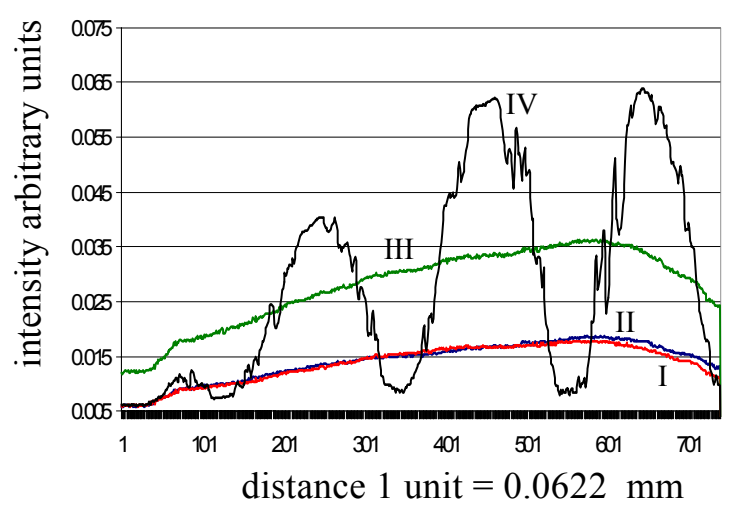

(c)

Figure 2: (a) Michelson interferometer arrangement. He-Ne laser is used as coherent light source. Light reflected from mirror 1 (M1) and mirror 2 (M2) are added together after going through beam splitters. Photograph of the interference pattern obtained from Michelson interferometer and its intensity profiles are also shown. (b) Non localized rectilinear fringes. (c) Intensity profiles of Michelson Interferometer. Curve $I$ (red): intensity from mirror $A$ only, curve $I I$ (blue): intensity from mirror $B$ only, Curve $I I I$ (green): algebraic sum (intensity $A+$ intensity $B$ ) curve $I V$ (black): intensity from both mirrors $A$ and $B$. The vertical axis is the output voltage of the photo diode amplifier that is proportional to the incident intensity of light and the horizontal axis (distance across the screen) is given by units ( 1 unit $=0.062 \mathrm{~mm}$ )

Results show that the intensities, $I_{1}$ and $I_{2}$ obtained for the separate beams are nearly equal within 97.4 percent. Light interference is observed when both beams are present, and the intensity relation at each maxima is $I_{\max } \approx 4 I_{1}$ within 98.2 percent. The area under the interference curve $I V$ is equal to the area under the curve $I I I$ or sum of curves $I$ and $I I$. At minima the intensity just exceeds the noise level. The results are in agreement with Eq. (5). This implies that the total energy of two light beams is equal to the energy of interference pattern and redistributed as shown in curve $I V$ in Fig. 2(c).

\subsection{This phenomenon can also be viewed from a different angle}

It is said that two wavefronts interact with each other and form a resultant wavefront, in which the energy is redistributed. The area under curve III (green) (mathematical summation of the intensities of two independent beams) and curve $I V$ (black) are 20.55 
(arbitrary units) and 20.92 (arbitrary units) respectively (Fig. 2(c)) and the difference is less than two percent. According to these results, it appears that the energy in the valleys has moved to the peaks of the curve $I V$.

The next study was planned to investigate whether the redistribution of energy that has been taken place within each beam, persists even after the two wavefronts have left the area where interference taken place. This experiment was carefully designed to overcome several drawbacks one would encounter when using a conventional experimental setup (Fig. 3(a)) with a source of electromagnetic wave in the visible region. One of the important aspects addressed at the design stage was the isolation of the two outgoing beams, after being interfered, to avoid any subsequent overlapping due to diffraction etc,. Conventional Young's double slit experiment setup (Fig. 3(a)) is not suitable for this purpose as the two immerging beams are nearly parallel and therefore bound to produce ambiguous results. A desirable separation of the two beams, of course, can be achieved if they are allowed to crossover with a large angle (Fig. 3(b)). Angle of crossover is independent of forming the minima because it is assumed that the $\vec{E}$ vector is always perpendicular to the direction of propagation

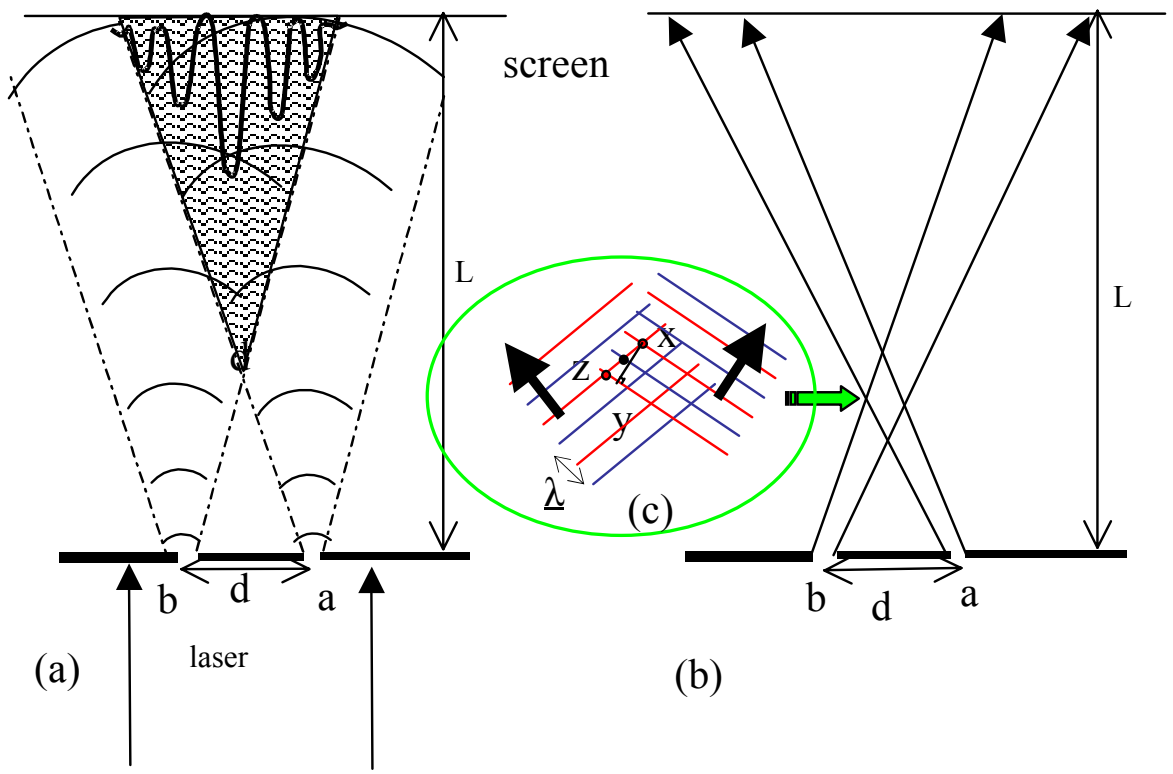

Figure 3: Young's double slit experiment and modified set-up. (a) Parallel laser light produces conventional young's interference pattern at a distance screen. (b) Two coherent electro magnetic waves cross each other and leaving the cross over zone separately. (c) How maxima are created when two electromagnetic waves are crossed each other with a large angle. $\boldsymbol{x} \boldsymbol{z}$ is the distance between two adjacent maxima. 
Once beams crossed each other with certain angle as shown in Fig. 3(c), two adjacent maxima are expected to be formed at points $x$ and $z$ where wave fronts interfere constructively. $\lambda$ is the wave length of the propagating wave. If the angle between the direction of propagation of the two waves is $\alpha$, then the distance $x z$ between the points $x$ and $z$ is given by

$x z=\frac{\lambda}{\operatorname{Sin} \alpha}$

For beams whose wavelength is in the visible region, for example He-Ne laser light of $\lambda=632 \mathrm{~nm}$ and crossing at right angle, the length $x z$ between two adjacent maxima is about $6.32 \times 10^{-7} \mathrm{~m}$. Currently a suitable detector is not available to measure such a small distance and detect maxima with a enough resolution, and therefore it is necessary to select two coherent beams of electromagnetic waves whose wave length is very much larger than that of visible light. However, in the experiment that was performed with the Michelson apparatus together with visible light, detection of the dark and bright fringes was possible as they were separated due to the fact that the two beams used were almost parallel $(\alpha \approx 0)$. see Fig. 2(b). For this reason, it was decided to carry out this experiment using microwave (band $\mathrm{X}$ - experimental band with centimeter wavelength region.) whose wave length is around $3 \mathrm{~cm}$. For this wavelength region, the value of $x z$ is around $3 \mathrm{~cm}$ when both beams cross each other at an angle of $90^{\circ}$, and currently available detectors should be capable of measuring intensity variation within such distance with good accuracy.

\subsection{Interference with Microwaves}

The experiment was carried out at the University of Manitoba, Canada and at the University of Moratuwa, Sri Lanka. Both experiments were performed in micro wave band X. An experimental set-up which uses BUDD STANLEY ED-SET (Model X4100, $9480 \mathrm{MHz}(\lambda=3.165 \mathrm{~cm})$ ) was used in Manitoba. A typical Klystron (JAN CS 2k25) based source was used as the microwave generator. Sivers Lab (Stockholm, Sweden) set-up and GUNN diode SGX12A (Toshiba) was used in Moratuwa.

The output power was split by a " $T$ " connector as shown in Fig. 4(a) and fed into two similar horn antennas. More accurate measurements were done in Moratuwa University 
under well-controlled conditions which were available at the microwave laboratory in the department of Electronics and Telecommunication. The mode of radiation of this horn antenna is TE10 having fairly less power in their side lobes. Few side lobes are also observed in this mode. For example, measured power ratio of first side lobe to the main lobe in these horn antennas is around $50 \mathrm{~dB}$. Two antennas were arranged in order to cross their main lobes at right angle and all the measurements were taken within the main lobes of respective antennas. The radiated power was measured by a PIN diode. The current induced in the detector is proportional to the incident power of microwave, and was measured by a digital microampere meter. The axes of output ports were set perpendicular to each other so that the radiated fields cross each other orthogonally. Two platforms $P Q$ and $R S$ were mounted as shown in Fig. 4(a) to receive separately the microwaves emitted from the antennas $A$ and $B$. Positioning of $P Q$ and $R S$ was done carefully so that they were outside the overlapping region of the two beams. The horn antenna with PIN detector was mounted on movable platform and the perpendicular distances from $C$ (the mid point of $A$ and $B$ ) to two platforms shown in blue and red (Fig. 4(a)) varied from $20 \mathrm{~cm}$ to $70 \mathrm{~cm}$ in Manitoba set-up. This distance was kept fixed at $20 \mathrm{~cm}$ (this distance is outside the crossover region) in Moratuwa experiment because the power output was found to be low compared to Manitoba set-up, and also a naked PIN diode was used here in order to measure total field strength induced by EM wave coming from all the directions. It seems that naked Diode detector gives a better estimate of the field strength at points to be measured in space. The actual detector size (less than in the order of $1 \mathrm{~mm}$ in diameter) and effective aperture were also small compared to the wave length. The distance between two radiators (center to center) was $10 \mathrm{~cm}$ in both experiments. For each platform, three sets of measurements were taken with antenna $A \mathrm{ON} B \mathrm{OFF}$, antenna $B \mathrm{ON}$ A OFF, and $A$ and $B$ both ON. When performing single antenna measurements, other end was closed with a suitable terminator.

\section{RESULTS AND DISCUSSION}

Fig. 4(b) shows the results of the measurement carried out in Moratuwa. The curve $I I$ (green) of Fig. 4(b) shows the intensity measured by the sliding detector along the platform $P Q$ when the antenna $A$ was $\mathrm{ON}$ and the antenna $B$ is properly terminated (OFF). As expected, the curve $I I$ gives the intensity pattern of a beam of microwave emanating from a 
single slit, within the main lobe of radiation pattern and which is more close to a bell shaped (close to Gaussian) distribution of a diffraction pattern. It is important to note that when the antenna $B$ was $\mathrm{ON}$ and $A$ was OFF, the intensity obtained from the sliding detector on platform $P Q$ was close to the noise level. See Fig. 4(b), curve $I I I$ (red). It clearly shows that the microwaves from antenna $B$ do not travel through the region where the platform $P Q$ is located.

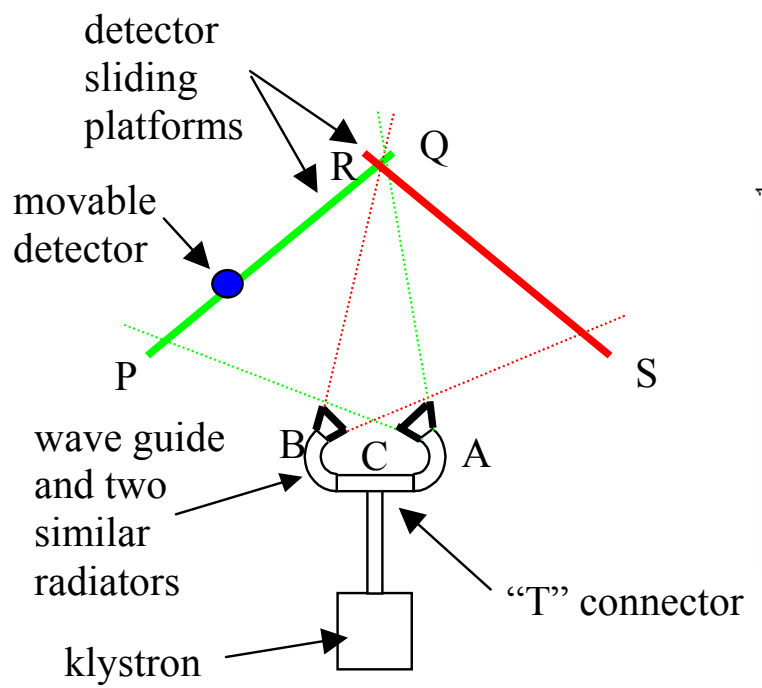

(a)

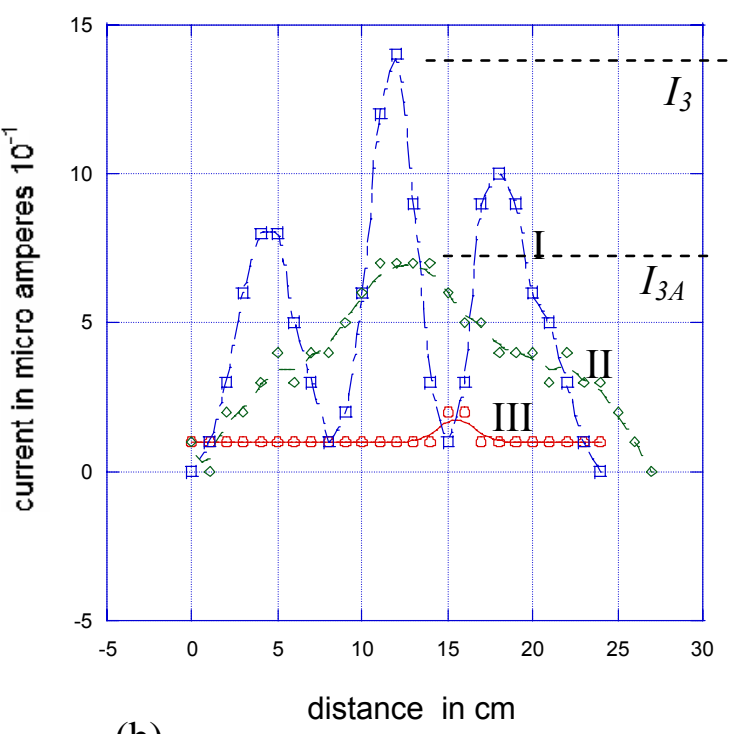

(b)

Figure 4: (a) Test set-up for observation of microwave interference. Arrangement is similar to the proposed experiment in Fig. 3(b). $A$ and $B$ are two horn antennas and are arranged in a way to obtain two microwave beams with equal intensities which cross orthogonally in space. $C$ is the mid point of $A B$. Sliding detector was mounted on $P Q$ and $R S$ which is outside the beam overlapping region. The perpendicular distance from $A$ to $P Q$ in this experiment is $20 \mathrm{~cm}$. (b) Microwave intensity profile obtained from the configuration in 4(a). $P Q$, Curve $I$-(blue) - Radiate from both antennas $A$ and $B$, Curve (II-green) - Radiation only from antenna $A$, Curve (III-red) - Radiation only from right antenna $B$. All the measurements were taken within the main lobe of Antenna $A$. Prominent interference is seen but almost all the power is received from antenna $A$.

However, when both antennas radiate power, the bell shaped intensity distribution turns in to a prominent interference pattern along the platform $P Q$ and that can be seen in curve $I$ (blue in Fig. 4(b)). Here the overall intensity relationship between before $\left(I_{3 A}\right)$ and after $\left(I_{3}\right)$ interference is close to $I_{3} \approx 2 I_{3 A}$ (see Fig. 6). Where $I_{3 A}$ is the measurement at $P Q$ when Antenna $A$ in ON and antenna $B$ is OFF. Similarly $I_{3}$ is the measurement at $P Q$ 
when both antennas are ON. The measured area under curve $I$ is 128 (arbitrary units), curve $I I$ is 100 units and curve $I I I$ is 26units. The algebraic sum of curves $I I$ and $I I I$ is 126 (arbitrary units), which is very close to measured area under Curve $I$ within 1.5 percent and that indicates the energy is conserved before and after interaction along PQ (where the measurements have been taken). Identical results were obtained for the $R S$ arm.

Each platforms practically receives most of the energy from its opposite antennas but still exhibit prominent interference patterns even though the platforms are out side the crossover region of the beams (see Fig. 4(a)). Note that the interference pattern appears in each beam only when the other beam crossed over.

Subsequently the platforms $P Q$ and $R S$ were moved away from antennas and, all the three measurements (antenna $A$ ON $B$ OFF, $B$ ON $A$ OFF and $A \& B$ both $\mathrm{ON}$ ) were taken at different distances $(20 \mathrm{~cm}$ to $70 \mathrm{~cm})$ from an antenna. The data in Manitoba is presented in Figure 5 as the intensity of the microwave source available at Moratuwa was not strong enough to carry out measurements at distance. The maximum distance was limited by the sensitivity of the sensor diode. The measurements show the same behavior as in previous results. Only differences observed were reduction of intensity and divergence of the shape of the interference pattern with the distance (Fig. 5(a)). Fig. 5(b) shows the enlarged view of the furthest observation at $70 \mathrm{~cm}$ from the antenna. The energy is propagated in a solid angle through space and therefore the reduction of energy with distance is expected. This observation clearly shows that each beam carried away 'the memory' of interference.

The interference pattern observed in each beam after the interaction of two waves cannot be explained using the classical interference theory. The only close explanation for a pattern like interference in a single beam is Fresnel diffraction but this is not possible due to two main reasons. Rayleigh and Fresnel fields (near fields) both exists within a distance $r$, from the antenna, where $r=\frac{2 L^{2}}{\lambda}$. Here $L$ is the length of the antenna (or the largest dimension of the aperture), $\lambda$ is the wavelength. The value of $r$ for the present case is 12.2 $\mathrm{cm}$ and it implies that the present measurements are well outside the near field zones. The experimental data shows that the shape of the interference pattern (after crossover region) has not changed with the distance, in the present case, it persists even at $70 \mathrm{~cm}$ from the antenna. See the inset of Fig. 5b. Fresnal diffraction is associated with a single slit ("near 
- field" interference). However, the interference that we observed in this experiment is produced by two crossed beams. In other words, this interference pattern not exists in the region between the antenna and crossover area when both antennas are $\mathrm{ON}$ but generates at the crossover and persists after that (see Fig. 6).

According to the classical theory the energy in the wavefront is redistributed only in the region of crossover. When the wavefront leaves the crossover zone, the energy redistribution should no longer be observed. However, in this experiment, interference patterns were observed even after the crossover. This clearly shows that the wavefronts which redistribute their energy at the crossover, retain the redistribution pattern even after leaving the crossover zone.

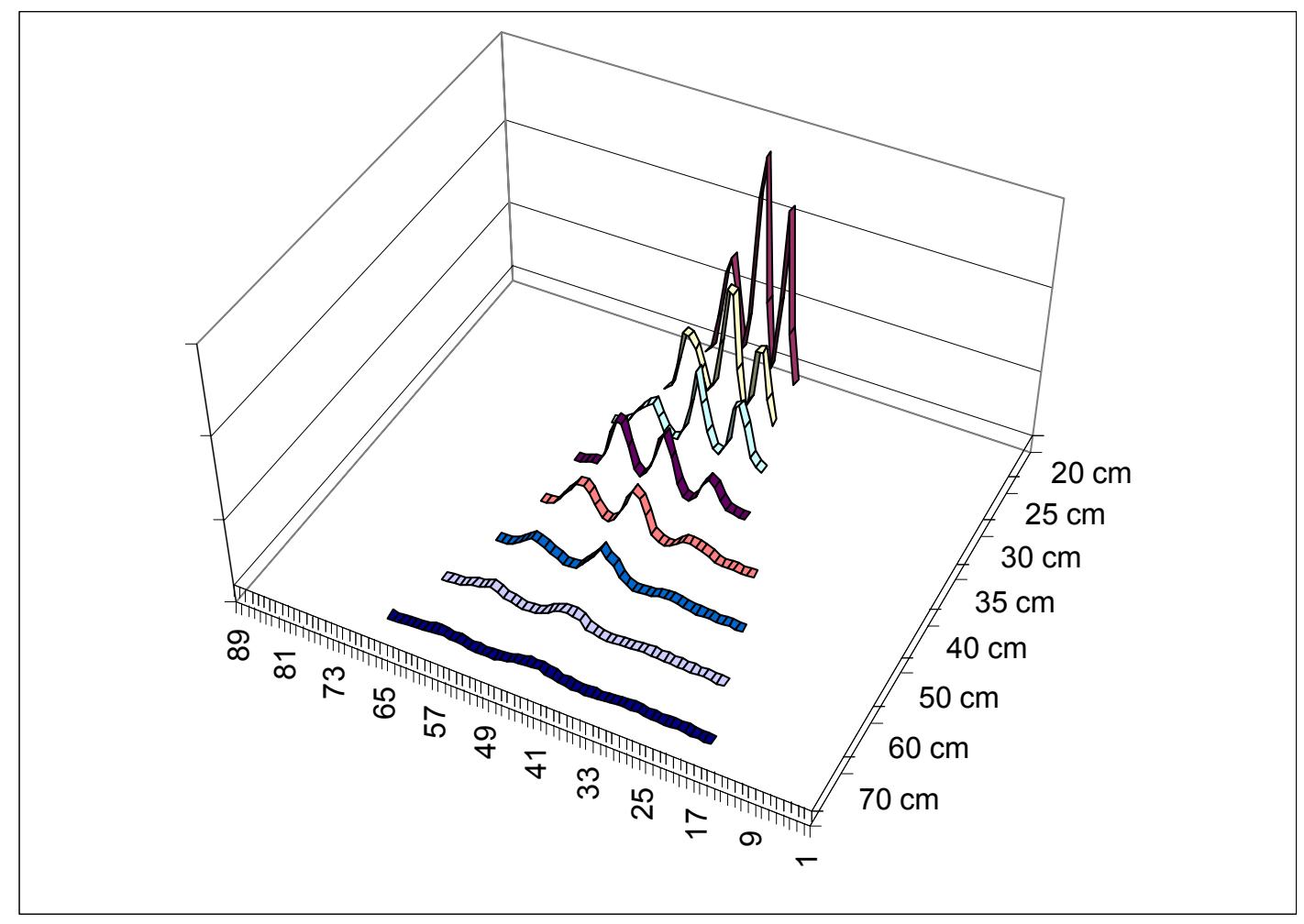

Figure 5: (a) Energy redistributed single beam after the crossover region. Intensity is gradually decreasing and beam shape is diverging with the distance. (b) Enlarged view of the intensity distribution of microwave at a distance $70 \mathrm{~cm}$ from the antenna.

Prior to the interference, each wave has uniformly distributed energy in the wavefront. When the two waves intersect, the wavefront is disturbed, resulting in new crests and troughs in each wavefront. The non-uniform energy distribution resulted due to the 
interaction of waves is retained as the two waves travel away from each other. However, the redistributed energy of each beam after crossover is equal to the energy of the original beam before crossover. This is clearly demonstrated by the experiment. If the energy distribution evens out after each wave moves away from the influence of the other wave, the interference pattern would not have been observed.

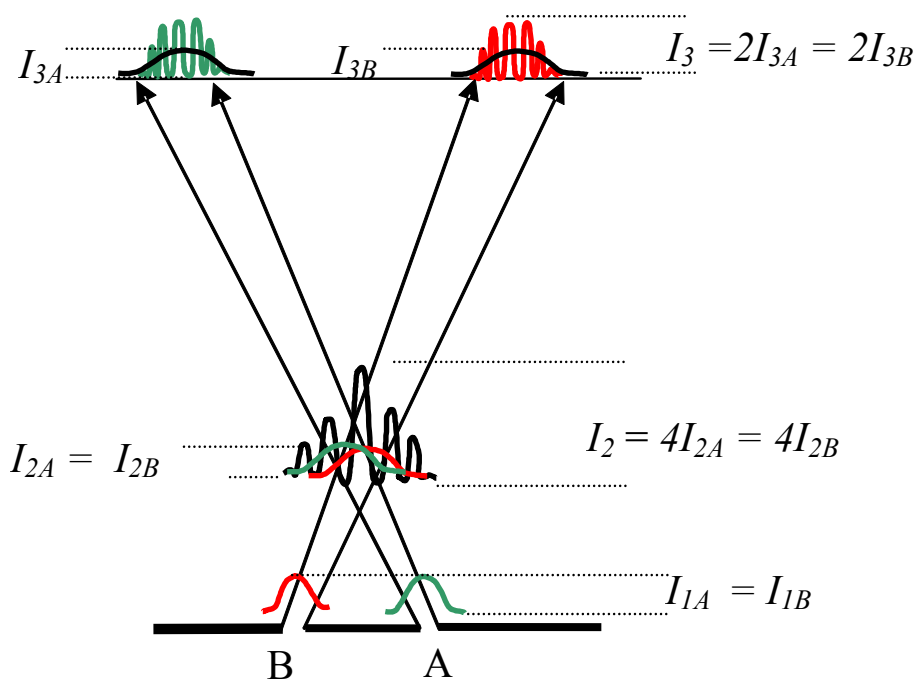

Figure 6: This figure pictorially summarizes the observations made in this experiment. After each antenna, usual bell shaped (close to Gaussian) distribution of a single slit diffraction is seen with a intensity $I_{I A}\left(I_{I B}\right)$. When two beams meet each other, interference occurs at crossover region with intensity $I_{2}=4 I_{2 A}\left(4 I_{2 B}\right)$. However, when two beams propagate after intersection each beam keeps its altered energy distribution which occurred due to interaction with other similar coherent wave at the crossover region. The relation of this intensity pattern is $I_{3}=2 I_{3 A}\left(2 I_{2 A}\right)$, which corresponds to the energy redistribution of a single beam.

Figure 6 pictorially summarize the observations made in this experiment. After each antenna, usual Gaussian or bell shaped distribution of a single slit diffraction exists with a certain intensities $I_{l A}$ and $I_{1 B}$ respectively for antenna A and B. When two beams meet each other, interference occurs with intensity $4 I_{2 A}$ (assuming that both beams have equal intensities). Where $I_{2 A}$ is the intensity of the bell shaped energy distribution of a single beam at the crossover region (when no second beam is presented). When two beams propagate after intersection, each beam keeps its altered energy distribution, which occurred due to interaction with other similar coherent wave at the crossover region. After the crossover region, the overall value of this intensity pattern measured at $P Q$ (which is similar to actual interference pattern) is $2 I_{3 A}$, which corresponds to the energy 
redistribution of a single beam. $I_{3 A}$ is the intensity of the bell shaped distribution of intensity of a single beam at $P Q$ when no second beam crosses at any point. This is similar to the situation in Fig. 4(a) ( $P Q$ and $R S$ arms) - microwave experiments where relationship $I=2 I_{1}$ holds.

\section{ACKNOWLEDGEMENT}

The Author gratefully acknowledges Dr. K. Samarasinghe, University of Moratuwa for providing laboratory facilities for this work, and Prof. T. R. Ariyaratna and Prof. N. De Silva for their valuable support and discussions. He also thank Prof. K. G. Standing. Prof. W. Ens, Prof. U Annakkage, Prof. Athula Rajapakse, Mr. V. Spicer, Mr. G. Roy and Mr. V. Pathirana for their support.

\section{REFERENCES}

1. L. Newton, OPTICKS or a treatise of the reflexions, refractions, inflexions and colours of LIGHT, Royal Society, London, (1704)

2. $\quad$ M. Planks, Analen der Physik, (1900-1901)

3. T. Young, On the Theory of Light and Colours, Philos. Trans, Roy. Soc., London 92, p1248, (1802)

4. C. Huygens, Traite de la Lumiere, Leiden: Van der Aa, (1690)

5. A. M. Michelson, Interference Phenomena in a new form of Refractometer, Am. J. Sci, (3), $\underline{\mathbf{2 3}}$, p395-400 (1882)

6. Fabry and Perot, Ann. Chimie Physique, 22, p564, Reprinted in Astrophys. Jour., 13, p265, (1901)

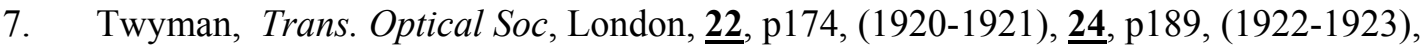

8. E. W. J. W. Mach, Modifikation und Anwendung des Jamin Interferenz-Refraktometers, Anz. Akad. Wiss. Wien math. Naturwiss.-Klasse, 28, p223-224, (1891); and L. Zehnder, Ein neuer Interferenzrefraktor, Z. Instrumentenkd, 11, p275-285, (1891)

9. A. J. Fresnel, Euvres, I, p330, (1816)

10. A. J. Fresnel, Euvres Completes, I, 150, p376, (1816)

11. Lloyd, Phys. Sc., Trans. R. Ir. Acad. XVII, p172, (1834) 